\title{
Système coutumier de tenure des terres et lutte contre la désertification en milieu rural au Burkina Faso
}

\author{
Souleymane Ouédraogo ${ }^{a}$, Marie-Claire Sorgho Millogo ${ }^{b}$ \\ a Agroéconomiste, INERA/CNRST, BP 476 Ouagadougou, Burkina Faso \\ b Sociologue, INERA/CNRST, BP 476 Ouagadougou, Burkina Faso
}

\begin{abstract}
Face à un environnement difficile, les sociétés sahéliennes développent des techniques d'aménagement qui peuvent entrer en contradiction avec le régime coutumier des terres, en particulier lorsque les populations d'origine doivent cohabiter avec les migrants. Le point de vue des auteurs défendant la flexibilité, dans certaines conditions, des régimes fonciers et des techniques d'aménagement ne fait pas l'unanimité, comme le rappellent les commentaires d'un géographe et d'un anthropologue. NSS rend donc compte de ce débat entre disciplines.
\end{abstract}

La Rédaction

\section{Mots-clés :}

système foncier ; conservation des sols ; ressources naturelles; désertification; Burkina Faso

Résumé - La question foncière a toujours été placée au cœur des problèmes de développement agricole au Burkina Faso et le système foncier coutumier, considéré par bon nombre d'acteurs comme une contrainte majeure à l'innovation. Cette étude s'inscrit dans une perspective de dynamique sociale en matière de gestion foncière et d'adoption de techniques de préservation des potentiels agricoles. Elle a été réalisée dans quatre villages de la région du Plateau central (Kirsi, Boulcon, Dafiré et Sakou) et dans deux villages de la zone ouest (Dossi et Bahoun). Le questionnaire administré auprès de 240 ménages et les outils MARP (méthode active de recherche participative) utilisés pour la collecte des données ont permis d'obtenir des informations qui montrent que le statut foncier (héritage, don, prêt à long terme, prêt à court terme et achat des terres) ne constitue plus un frein à des investissements agraires sur le long terme. Dans le registre des techniques de conservation des ressources naturelles (cordons pierreux, zaï, demi-lune, bandes enherbées, paillage, plantation d'arbres, épandage de fumier, haie vive), seule la plantation d'arbres semble encore ne pas être acceptée par les possesseurs terriens.

\section{Keywords:}

land tenure; customary systems; land status; technologies; desertification; Burkina Faso

\begin{abstract}
Customary land tenure system and combating desertification in rural areas of Burkina Faso. Land tenure has always been at the heart of agricultural development issues in Burkina, while the customary system has been considered by a great number of actors as one of the major constraints to adoption of improved land management techniques. Our study examines the social dynamics of land management and adoption of land management techniques to preserve agricultural potential against desertification.

The study was carried out in four villages of the Central Plateau zone (Kirsi, Boulcon, Dafiré and Sakou) and in two villages of the West zone (Dossi and Bahoun) and included 240 households. The information obtained from the data collected using MARP (Méthode Active de Recherche Participative) tools showed that land status (land inheritance, gift, long-term loan, short-term loan and purchase) is no longer a constraint to adopting long-term land conservation techniques. Among the resource conservation methods practiced in the villages (stone rows, zais and half moon basins, grassed strips, mulching, tree planting, manure spreading, quickset hedges) only tree planting does not yet seem to be accepted by landowners.
\end{abstract}

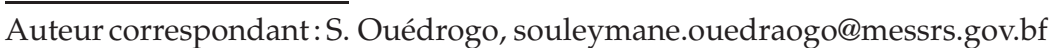




\section{Introduction}

Les problèmes associés à la gestion des ressources naturelles, en Afrique en particulier, prennent une place de plus en plus importante dans les préoccupations des décideurs politiques. Au cours des deux dernières décennies, les communautés rurales du Burkina Faso, pays agricole à la lisière du Sahel en Afrique de l'Ouest, ont connu des changements et des stress importants. Une pluviométrie plus faible et un accroissement démographique rapide se sont traduits par une dégradation des terres cultivées (érosion éolienne et hydrique, appauvrissement en matière organique et en nutriments, disparition d'arbres et $d$ 'arbustes) entraînant une baisse des rendements agricoles. Les paysans y font face en cultivant plus de terres, ce qui se fait aux dépens des jachères, accélérant ainsi la baisse de fertilité. En conséquence, on assiste à l'augmentation des conflits entre agriculteurs et éleveurs et à une pression de plus en plus élevée sur les parcours et les terres communes.

Au-delà des problèmes liés à la croissance de la pression démographique, phénomène contre lequel on ne peut rien à court terme, la dégradation des ressources naturelles est souvent attribuée au système coutumier de gestion commune des ressources (Runge, 1992). On reproche à ce dernier de ne pas s'inscrire dans une logique économique et d'être soumis à des contraintes socioéconomiques et culturelles qui contribuent à la dégradation des écosystèmes. En effet, il semble que toute innovation technique ou socioéconomique qui risque de mettre en cause l'organisation de la société se heurte à une résistance sociale.

$\mathrm{Au}$ Burkina Faso, la question foncière a toujours été au centre du développement agricole (Boutillier, 1964; Kohler, 1971; Mesnil, 1970; Ouédraogo, 1993). Selon l'idée la plus répandue, le système foncier coutumier constitue une contrainte majeure à la modernisation de l'agriculture. Il a été longtemps considéré comme un obstacle à l'investissement. Boutillier (1964) écrivait déjà que, dans «le droit foncier voltaïque, droit collectif et droit individuel s'enchevêtrent et l'on peut penser que devant l'instabilité de leur titre foncier, des cultivateurs progressistes hésiteraient à entreprendre des améliorations foncières qui relèveraient la valeur de la terre ». Pour Ouédraogo (1993), les coutumes foncières s'opposent aux investissements créateurs ou les découragent. Elles confinent les exploitants à l'autosubsistance et interdisent les travaux d'amélioration, d'entretien et de plantation d'arbres. Un système foncier «fonctionnel » serait indispensable pour améliorer l'efficacité de la production agricole et la diversification de l'utilisation des terres. C'est fort de cette conviction que le Conseil national de la révolution (CNR) a promulgué la réorganisation agraire et foncière en 1985. L'objectif visé était de briser les contraintes imposées par la gestion coutumière du foncier afin d'accélérer la croissance économique. Cette réorganisation, malgré de multiples relectures pour l'adapter aux conditions du monde rural, n'a pu être effectivement appliquée. C'est dire que la question foncière a toujours été un objectif majeur des politiques au Burkina Faso. Elle reste cependant très controversée. Le débat est ouvert entre les partisans d'une privatisation rapide, susceptible d'accélérer l'investissement, et ceux qui pensent qu'il faut se contenter de suivre une tendance naturelle progressive vers cette privatisation. Ce qui est sûr, c'est que les institutions ou les mécanismes qui contrôlent l'utilisation et l'allocation des ressources doivent s'adapter à un contexte socioéconomique en évolution rapide. En effet, dans la situation actuelle marquée par la dégradation de l'environnement et le besoin d'assurer la sécurité alimentaire, le souci de plus en plus répandu des populations est d'investir dans l'amélioration des terres et la lutte contre la désertification.

Dans ce contexte, on peut se demander si la dynamique des changements en cours dans le milieu rural a eu raison des règles coutumières de gestion des terres. En d'autres termes, le système foncier coutumier constitue$\mathrm{t}$-il encore une contrainte aux investissements agricoles, particulièrement à l'adoption des techniques de lutte contre la désertification? Pour répondre à cette question, une enquête a été menée dans six villages du Burkina Faso afin d'identifier les techniques de lutte contre la désertification en cours et le degré de leur mise en œuvre en rapport avec le statut foncier ${ }^{1}$.

\section{Régime foncier et développement}

Le système foncier coutumier a toujours été au centre de nombreuses controverses. La grande majorité des économistes (surtout les économistes néoclassiques) mettent en doute son efficacité économique et écologique. Il serait à l'origine de la piètre performance de l'agriculture en Afrique subsaharienne. Les principaux arguments avancés par ces économistes sont qu'il n'encourage pas l'investissement (Feder, 1987; Feder et Feeny, 1991; Binswanger et al., 1995; Famorio et Adéniyi, cités par Francis, 1986; Bruce, 1985; Curie, 1981; Mellor, 1966). Ce régime foncier coutumier est donc incompatible avec le développement. Les auteurs cités soutiennent que la structure du régime foncier empêche la mobilisation des ressources productives. De plus, ce système entrave l'accès à la terre pour des agriculteurs extérieurs au groupe

\footnotetext{
${ }^{1}$ Ce travail s'inscrit dans la thématique de la recherche sur le système foncier que mène le département Gestion des ressources naturelles de l'Institut de l'environnement et des recherches agricoles (INERA). Il sera présenté au mois de mai 2007 lors d'un colloque sur la désertification, en cours de préparation par l'IRD et le Desert Margin Programm (DMP), qui aura lieu à Ouagadougou (Burkina Faso).
} 
possesseur de la terre. Les auteurs attribuent les problèmes précédents à la tenure communautaire et au système coutumier de faire-valoir. Pour eux, la modernisation de l'agriculture passe par la création d'unités économiques viables, l'investissement dans les exploitations. Or, l'acceptation de l'investissement et la recherche de la rentabilité requièrent que les agriculteurs se sentent responsables de leur terre (Lavigne Delville et al., 2001) ${ }^{2}$, ce qui n'est pas le cas avec les modes coutumiers d'occupation et leur système de redistribution périodique des terres. Des économistes considèrent aussi que les pesanteurs des modes coutumiers de tenure foncière bloquent l'accroissement de la productivité par hectare et par exploitant.

Or, cette vision est contestée de nos jours. De nombreuses études ont montré que, contrairement à ce que pensent les économistes classiques, le système foncier coutumier est flexible, dynamique et s'adapte aux changements (Bassett, 1993 ; Bruce et al., 1994 ; Platteau, 1996 et 1998 ; Mathieu, 1995), et que la corrélation entre la sécurité foncière et l'investissement n'est pas clairement établie. Il en est de même de la sécurité foncière et de la productivité (Biaou, 1991; Ouédraogo, 1991; MigotAdholla et al., 1993; Ouédraogo et al., 1996; Sjaastad et Bromley, 1997). Les théories évolutionnistes des droits fonciers suggèrent quant à elles que le système foncier coutumier est efficace en situation de faible pression démographique, mais devient obsolète dès que la pression augmente sur les ressources naturelles. Elles affirment que la pression démographique croissante conjuguée à l'économie de marché crée un besoin d'investissement et pousse à modifier les régimes fonciers.

Une lecture historique de l'évolution des règles d'accès à la terre semble donner raison à ce second courant de pensée. L'observation de l'environnement (institutionnel, économique, social et politique) montre qu'au Burkina Faso, les arrangements fonciers ont évolué sous l'action de certains facteurs (pression de la population sur le foncier, économie monétaire, individualisme économique, actions des ONG et des projets de développement) et ont contribué à apporter des changements qui ont déstabilisé les structures coutumières de gestion du foncier. Dans bon nombre de cas, ils évoluent de façon à donner plus de droit à l'individu.

\section{Régions étudiées et méthodologie}

\section{Le Plateau central}

Le Plateau central est une vaste étendue de terres de $70668 \mathrm{~km}^{2}$ (soit $25 \%$ du territoire national) couvrant la partie centrale du Burkina Faso, à cheval entre

\footnotetext{
${ }^{2}$ Ces auteurs posent, eux aussi, le principe initial que « les ruraux ont besoin d'une sécurité foncière suffisante pour pouvoir produire de façon efficace ».
}

les zones nord-soudanienne et subsahélienne. C'est une entité géographique où les sols sont en général peu profonds, pauvres en éléments nutritifs principaux et d'une faible capacité de rétention en eau. Pourtant, c'est la région la plus peuplée du Burkina Faso. Sa population est passée de 3584000 habitants en 1985 à 4879000 en 1996 - soit un taux d'accroissement annuel moyen de $3 \%$-, ce qui représente $48 \%$ de la population totale du pays. Là, se trouvent les principales villes du pays dont la capitale Ouagadougou, qui compte environ 1000000 d'habitants. La population relève pour la plupart du groupe ethnique Mossi, qui cohabite harmonieusement avec les Gourounsi à l'ouest et les Bissa au sud-est. La densité moyenne de la population est de $50 \mathrm{hab} / \mathrm{km}^{2}$. Cette moyenne cache cependant d'importantes variations régionales (28 hab $/ \mathrm{km}^{2}$ dans la province du Namentenga, dans le centre-nord, en 1985 contre $88 \mathrm{hab} / \mathrm{km}^{2}$ dans le Kouritenga au centre-est et 391 dans le Kadiogo, dans le centre, où se situe Ouagadougou).

Avec les ressources en terres actuellement disponibles, le Plateau central ne devrait pas avoir une densité de population supérieure à $40 \mathrm{hab} / \mathrm{km}^{2}$. On estime que le seuil agrodémographique ${ }^{3}$ de l'utilisation des terres de cette partie du pays est dépassé, avec, comme conséquence, une dégradation avancée des ressources naturelles. Le constat d'une érosion grandissante a été fait au Yatenga dès les années 1960. Depuis lors, lutte antiérosive, reboisement, reconstitution du pâturage et enfin mise en défens des sols sont affichés comme des programmes prioritaires.

La forte concentration de la population sur le Plateau central n'est pas sans poser des problèmes dans la mesure où plusieurs maux lui sont imputables. Elle contraste avec les potentialités agricoles de la région. En 1985, la superficie agricole utile était de $28350 \mathrm{~km}^{2}$, soit $30 \%$ des terres arables du pays, ce qui représentait une superficie agricole utile de $0,94 \mathrm{ha} / \mathrm{hab}$ en moyenne et un taux d'intensité culturale de $47 \%$. Au fur et à mesure de l'accroissement de la population, des terres marginales sont mises en culture. Si, à cela, l'on ajoute les conditions climatiques et pluviométriques souvent peu favorables et des méthodes encore extensives d'exploitation des sols, le Plateau central éprouve de sérieuses difficultés pour satisfaire les besoins alimentaires de sa population. Face à cette situation et dans le souci d'assurer leur subsistance, les populations ont développé au fil des années des techniques simples pour pallier l'insuffisance des productions agricoles. Ces différentes techniques ont été décrites par plusieurs auteurs dont Vlaar (1992), Rochette (1989), Marchal (1986), Reij (1983) et Roose (1994).

\footnotetext{
${ }^{3}$ Le seuil agrodémographique renvoie à la relation entre superficie en jachère et superficie en culture. En dessous de ce seuil, la fertilité des terres exploitées en cultures extensives n'est plus régénérée.
} 


\section{La région ouest}

Elle est reconnue comme la première région agricole du Burkina Faso. Située entre les isohyètes 700 et $1200 \mathrm{~mm}$, elle a un potentiel agro-sylvo-pastoral important, ce qui permet une gamme variée de productions. La culture du coton, introduite par le colonisateur français et réhabilitée par les pouvoirs publics depuis l'indépendance du pays en 1960, a entraîné des innovations techniques et sociales. C'est la région qui possède le meilleur taux d'équipement en matériel agricole (40\% des exploitations).

Avec moins de $30 \%$ de la population, 1'Ouest dispose de $35 \%$ des surfaces arables du pays. L'environnement démographique, eu égard à l'étendue de la région, n'est pas homogène. La population relève des groupes ethniques Bwa, Sénoufo, Mossi, Lobi et Dagara. Les densités de population sont faibles, ainsi que le taux d'intensité culturale. La superficie agricole par habitant varie de 1,5 à 4 ha. L'abondance des terres agricoles est l'un des facteurs qui a suscité l'intérêt des migrants pour la région. Leur installation s'est faite selon les mécanismes coutumiers d'insertion des étrangers, l'attribution des terres étant individuelle ou collective. Dans le premier cas, les premiers arrivés se voyaient attribuer de vastes étendues de terres et ils pouvaient à leur tour en céder des portions à d'autres venus. Dans le second cas, les autochtones attribuaient un secteur à un ensemble de migrants, lesquels devaient en assurer eux-mêmes la répartition. Les facilités offertes aux premiers migrants ont stimulé l'ensemble du mouvement migratoire. C'est cette poussée démographique qui est à l'origine de la pression actuelle sur les ressources naturelles. Il en a résulté une augmentation des superficies cultivées, une réduction de la durée de la jachère, une dégradation des terres, un changement de comportement des autochtones par rapport à l'exploitation de leur domaine foncier, une course à la terre provoquée par les migrants.

\section{Le régime foncier coutumier}

Le Burkina Faso peut être considéré comme homogène sur le plan du régime foncier. Sur toute l'étendue du territoire, sauf dans la partie sahélienne, on note l'existence d'un chef de terre, généralement descendant du lignage du groupe des premiers occupants de la terre. Il assure les fonctions religieuses et juridiques et gère la terre pour le compte de la collectivité. Il est l'autorité compétente en matière foncière. C'est lui qui procède à la redistribution périodique des terres aux membres de la collectivité et aux étrangers, en tenant compte des besoins de chacun. En procédant de la sorte, il évite l'accaparement et la sous-utilisation des terres. Si, dans le Plateau central, le chef de terre est différent de celui du village, dans l'Ouest, il arrive que le chef de terre soit en même temps le chef de village. Là où les deux fonctions sont séparées, le chef de terre rend compte au chef de village.

Ce régime foncier se fondait initialement sur une conception de la relation entre l'homme et la terre. Il reposait sur le principe qu'il existe pour chaque homme un droit naturel à la terre, c'est-à-dire le droit pour tout individu, qu'il appartienne à la communauté ou non, de disposer d'une parcelle pour assurer sa subsistance. Au sein du village, chaque groupe familial a un droit d'usage sur une partie du patrimoine foncier dont l'étendue est fonction des besoins. Si un membre du groupe dispose d'assez de terres, il est moralement obligé d'en donner ou d'en prêter une partie à quiconque en fait la demande. Deux types de droit d'usage sont reconnus par le régime foncier coutumier :

- un droit d'usage permanent dévolu à tout membre de la famille étendue, du lignage ou du groupe qui partage l'appropriation collective des terres. La distribution des terres est assurée par l'aîné du groupe, qui joue le rôle de gérant du patrimoine foncier lignager ; - un droit d'usage temporaire accordé généralement à tout individu extérieur au groupe sous forme de prêt. En principe, ce droit s'estompe avec l'arrêt de la mise en culture de la parcelle concédée. Mais ce droit inhérent au prêt de terre est assorti d'obligations dans la plupart des communautés. Il interdit à l'emprunteur de réaliser des ouvrages considérés comme signes d'appropriation de la terre, tels que la plantation d'arbres, le forage de puits, etc.

Ce schéma de fonctionnement du système foncier cadrait bien avec une agriculture de subsistance et avec une faible pression sur les ressources. Cependant, l'accroissement de la population et l'évolution économique et institutionnelle ont favorisé des unités familiales restreintes et ont, de ce fait, entraîné des perturbations dans les pratiques foncières, notamment dans les conditions d'accès à la terre. Une certaine rigidité dans la transmission des droits s'est substituée à la possibilité de redistribution des terres lignagères, particulièrement dans les zones densément peuplées comme le Plateau central. Comme le notait Kohler (1971), « le lot des terres communes dont l'usage était soumis au contrôle permanent et direct de l'aîné du lignage a été progressivement partagé en parcelles et leur usage est devenu un droit héréditaire strict à l'intérieur des lignages ». La tendance actuelle est à subordonner de manière permanente les droits d'usage à ceux de possession.

\section{Choix des villages et collecte des données}

Les six villages enquêtés ont été choisis de concert avec les structures de développement et des ONG qui travaillent dans les deux régions. L'hypothèse de notre 
recherche étant d'évaluer l'impact du système foncier coutumier sur la mise en œuvre des techniques de lutte contre la désertification, le choix s'est porté sur des villages où il existe une dynamique foncière en cours et qui ont bénéficié d'un accompagnement de la part des ONG et des projets de développement, particulièrement dans le domaine de la lutte contre la désertification et, d'une manière plus large, dans celui de l'intensification de la production agricole.

D'après les critères ci-dessus, tous les villages du Plateau central étaient éligibles dans la mesure où cette région présente des conditions idéales pour ce genre de recherche. En effet, depuis les années 1970, on assiste à une floraison d'ONG et de projets de développement, en raison du niveau exceptionnel de dégradation des écosystèmes, accentué par des sécheresses récurrentes, et du succès incontestable des actions de lutte contre la désertification. Notre choix s'est cependant porté sur Boulkon, Kirsi, Sakou et Dafiré. Ces quatre villages sont encadrés depuis les années 1990 par le projet Conservation des eaux et des sols - Agroforesterie (CES/AGF) et le Projet d'aménagement des terroirs et de conservation des ressources (PATECORE), tous deux dans le Plateau central.

La même démarche a été suivie pour le choix des deux villages de l'Ouest. Contrairement au Plateau central, le niveau de dégradation des terres et les interventions des ONG et des projets demeurent faibles. Le choix s'est porté sur Dossi et Bahoun, dans la province du Tuy. Ces villages ont pendant longtemps accueilli un important flux migratoire d'agriculteurs du Plateau central, qui s'est ralenti ces dernières années. L'installation des migrants s'est accompagnée d'un recul des règles coutumières de gestion de l'espace, qui devient plus effective à l'échelle des lignages que du chef de terre. Si la transmission des droits par les autochtones aux migrants de la première génération n'est pas remise en cause, celle concédée par ces derniers aux migrants de la seconde génération l'est en grande partie. Dès lors, des pratiques proscrites par le droit coutumier (par exemple, le refus d'octroyer de la terre à un étranger) se développent aujourd'hui. La situation actuelle des autochtones se caractérise par une liberté d'initiative sans recours à l'autorisation du chef de terre. Elle est consommatrice d'espace et constitue une cause importante de la dégradation des terres.

Deux types d'outils de collecte des données ont été utilisés :

- un questionnaire structuré administré auprès d'un échantillon de 40 ménages choisis de façon aléatoire dans chaque village, soit un total de 240 ménages - ce qui représente à peu près 5 à $10 \%$ des ménages des villages concernés. Le choix aléatoire a été privilégié de façon à pouvoir classer les ménages en fonction de leur statut foncier et de se faire ainsi une idée de leur représentativité ;
- des outils de la méthode active de recherche participative (MARP), notamment l'interview semi-structuré, l'historique et la carte du village, ceci afin d'engager la discussion avec l'ensemble des ménages sur les changements qui sont intervenus dans la gestion foncière. Ces discussions ont été réalisées à l'échelle des villages et en groupe (hommes, femmes, jeunes) pour permettre la triangulation de l'information.

\section{Présentation des statuts fonciers et des techniques de lutte contre la désertification}

Deux types de statuts fonciers existent en milieu rural burkinabé : les possesseurs fonciers et les nonpossesseurs. Les premiers disposent d'un droit d'usage permanent, alors que les seconds ne bénéficient que d'un droit d'usage temporaire.

\section{Les possesseurs fonciers}

Ce sont des autochtones qui ont accédé à la terre par héritage. Font partie également de ce groupe, ceux qui ont bénéficié d'un droit d'usage permanent par donation (droit de hache ou de défrichage) ou par achat.

- L'héritage reste la principale forme d'accès à la terre. Dans sa forme originelle, l'héritage se faisait à l'intérieur du lignage premier occupant de la terre. Cependant, avec l'évolution récente, l'héritage tend à passer de père en fils. Au sein des ménages enquêtés, l'ensemble des autochtones cultive sur des terres héritées. Les normes sociales et la cohésion familiale leur garantissent le droit de jouissance. L'héritier ne peut être exproprié.

- Le don : par cette forme de cession, la communauté autochtone se «dessaisit», sous conditions de respect des coutumes du village, d'une portion de son terroir au profit d'un étranger ou d'un groupe d'étrangers qui s'y installent. Le don peut être interprété comme une forme de détournement de la règle de l'inaliénabilité de la terre dans les coutumes africaines. Ce sont généralement les chefs de terre et de village qui sont à l'origine de cette forme de cession. Elle confère un droit de propriété aux bénéficiaires, droit qui est irrévocable et se transmet d'une génération à une autre. À ce titre, les bénéficiaires sont intégrés dans la communauté et jouissent d'un droit d'usage permanent identique à celui des autochtones sur la portion qui leur est cédée.

- L'achat des terres : il exprime une transformation profonde du rapport homme-terre. La terre n'est plus inaliénable, mais fait désormais l'objet de transactions monétaires. L'achat de terres est le fait d'agriculteurs 
et de commerçants désireux de pratiquer l'arboriculture ou l'élevage. Cette forme d'accès à la terre se rencontre surtout dans les zones périurbaines et très rarement en milieu rural.

Dans ce type de statut, la presque totalité des terres constitue la propriété du ménage et elles ne peuvent lui être retirées. En plus de celles qui lui appartiennent, il peut également exploiter des terres sur lesquelles il ne détient qu'un droit d'usage temporaire. Cependant, il jouit dans l'ensemble d'une sécurité foncière.

\section{Les non-possesseurs fonciers}

Ils ne détiennent pas des droits sûrs et intangibles sur la plus grande partie des terres qu'ils cultivent. C'est le cas de ceux qui ont accédé à la terre par des contrats de prêt à court ou à long terme.

- Le prêt à long terme : c'est la principale forme de cession des terres aux étrangers. Le droit d'usage est quasi permanent, mais tacitement renouvelé chaque année, ce qui laisse entrevoir la possibilité pour le prêteur de retirer son terrain. De par son caractère indéfini, ce type de prêt se confond souvent avec le don, mais il s'en distingue par le fait que la notion de délai n'intervient pas au moment de la cession en ce qui concerne le don. De plus, le don offre un droit d'usage permanent, alors que le prêt à long terme donne un droit d'usage quasi permanent.

- Le prêt à court terme : il est né de la raréfaction des terres. Par cet accord, les ménages bénéficiaires ne jouissent que d'un droit d'usage précaire. Le prêt à court terme traduit des difficultés d'installation et une situation de blocage foncier.

Les producteurs qui entrent dans cette catégorie peuvent être propriétaires d'une petite portion des superficies qu'ils exploitent (champs de case), mais la majeure partie relève $\mathrm{du}$ village ou d'autrui. Ils sont le plus souvent en situation de précarité, les terres pouvant leur être retirées chaque année. Coutumièrement, les contrats qui les lient aux possesseurs ne leur permettent ni de planter des arbres ni d'entreprendre des améliorations foncières qui pourraient être considérées comme une affirmation de leur droit. Cette restriction permettait de sécuriser les possesseurs. En effet, la marque du travail était considérée comme une forme d'appropriation; de ce fait, planter des arbres ou investir dans l'amélioration de la terre était anticipé comme une tentative de la part de l'emprunteur de s'approprier définitivement la terre. Tout manquement à ces interdits pouvait alors conduire au retrait du champ.
Tableau 1. Répartition des ménages enquêtés en fonction du statut foncier.

\begin{tabular}{|c|c|c|c|c|c|}
\hline \multirow[t]{2}{*}{ Village } & \multicolumn{2}{|c|}{ Possesseurs terriens } & \multicolumn{2}{|c|}{ Non-possesseurs terriens } & \multirow[t]{2}{*}{ Total } \\
\hline & Nombre & $\%$ & Nombre & $\%$ & \\
\hline Kirsi & 25 & 62,5 & 15 & 37,5 & 40 \\
\hline Boulcon & 4 & 10,0 & 36 & 90,0 & 40 \\
\hline Dafiré & 22 & 56,4 & 17 & 43,6 & 39 \\
\hline Sakou & 19 & 47,5 & 21 & 52,5 & 40 \\
\hline Dossi & 10 & 32,3 & 21 & 67,7 & 31 \\
\hline Bahoun & 10 & 32,3 & 21 & 67,7 & 31 \\
\hline Total & 90 & 40,7 & 131 & 59,3 & 221 \\
\hline
\end{tabular}

\section{Répartition des ménages en fonction du statut foncier}

Le tableau 1 indique la répartition des ménages des six villages en fonction du statut foncier. Il ressort d'une manière générale que la proportion des non-possesseurs dépasse de loin celle des possesseurs dans presque tous les villages, mis à part ceux de Kirsi et de Dafiré.

Pour les quatre villages du Plateau central (Kirsi, Boulcon, Dafiré et Sakou), 44 \% des ménages en moyenne sont possesseurs de leurs terres et $56 \%$ non-possesseurs. Ces chiffres étaient respectivement de $67 \%$ et $33 \%$ en 1964. On assiste donc à une inversion, avec l'accroissement de la population et la pression sur le foncier. Cette inversion révèle clairement les mutations et les transformations sociales en cours dans le milieu rural. C'est d'ailleurs ce qu'avait anticipé Boutillier (1964), lorsqu'il écrivait qu' « au fur et à mesure que la densité croît, c'està-dire que la terre se fait de plus en plus rare, la tenure se fait de moins en moins souple; les règles de dévolution successorale deviennent plus rigides, ce qui a pour effet de faire baisser la proportion des champs hérités et d'usage permanent hérité et de faire monter la proportion des champs prêtés ". L'évolution des chiffres peut également traduire un morcellement accru des terres lignagères ou familiales (fractionnement des champs entre héritiers), de sorte que les héritiers de certains ménages qui disposaient du droit d'usage permanent se retrouvent dans une situation où ils doivent recourir à des prêts pour satisfaire leurs besoins. En effet, l'accès à la terre par les nouveaux ménages ne se fait plus uniquement à partir du partage des seules terres lignagères, mais également par des emprunts à d'autres lignages.

La tendance est identique dans les deux villages de l'Ouest (Dossi et Bahoun) : $32 \%$ des ménages sont des autochtones possesseurs de la terre contre $68 \%$ n'ayant que des droits d'usage temporaires. Si, dans le Plateau central, ces chiffres s'expliquent par l'accroissement naturel de la population, dans l'Ouest, ils traduisent l'importance de la migration. En effet, la souplesse du droit foncier coutumier bwa a favorisé l'installation de nombreux ménages venus d'ailleurs, principalement du Plateau central. Ce qui fait que, de nos jours, la plupart des 
villages bwa comptent plus de familles allochtones que d'autochtones.

\section{Les techniques de lutte contre la désertification}

La dégradation des terres suite à l'accroissement de la population a fini par créer un besoin d'investissement dans la conservation et l'amélioration des ressources naturelles. On estime que, sur le Plateau central, les techniques de conservation des eaux et des sols (CES) couvrent 250000 à 300000 ha. Dans l'Ouest, par contre, les superficies aménagées sont nettement moindres en raison du faible niveau de dégradation des ressources naturelles. Les techniques de lutte contre la désertification utilisées par les paysans varient en fonction des écosystèmes et des conditions socioéconomiques des ménages, notamment leurs capacités à disposer de la maind'œuvre suffisante et des moyens matériels, tel le petit équipement agricole (Rochette, 1989). En plus de leurs pratiques propres, les paysans adoptent des techniques améliorées vulgarisées, basées sur leur savoir-faire. La mise en œuvre de ces techniques vise trois objectifs : conserver et protéger la terre; améliorer la fertilité ; accroître la production agricole.

Afin de comprendre le rôle joué par la tenure foncière, nous avons, dans un premier temps, recensé les techniques de lutte contre la désertification qui sont appliquées dans les villages. Neuf principales techniques ont été dénombrées dans le Plateau central : cordons pierreux, zaï, demi-lune, régénération naturelle assistée, plantation d'arbres, bande enherbée, fumure organique, paillage et haie vive (Photos) contre sept dans la région ouest : cordons pierreux, régénération naturelle assistée, plantation d'arbres, paillage, bande enherbée, fumure organique et haie vive. Ces techniques n'ont cependant pas le même niveau d'impact. Le producteur fait son choix en se fondant sur un certain nombre de critères qui sont, entre autres, les impacts sur le rendement ou la productivité du sol, sur sa fertilité, l'humidité du sol et la durabilité des ouvrages. Ces critères prennent en compte simultanément des objectifs à court et moyen terme (rendement, fertilité) et d'autres à long terme (durabilité).

- Les cordons pierreux : ce sont des barrières mécaniques d'arrêt ou de freinage des eaux de ruissellement placées le long des courbes de niveau, pour réduire l'érosion et augmenter le stock d'humidité du sol. Les pierres sont disposées dans des tranchées de 10 à $15 \mathrm{~cm}$ de profondeur. La largeur d'un cordon est d'environ 15 à $20 \mathrm{~cm}$ et la longueur varie entre 10 et $30 \mathrm{~m}$. La disponibilité en main-d'œuvre et la présence de cailloux constituent les facteurs limitants. Les cordons pierreux entraînent une réduction du ruissellement, facilitent l'infiltration de l'eau dans le sol et favorisent une accumulation des particules en amont des diguettes. L'état hydrique du sol permet aux cultures de supporter des épisodes de sécheresse et d'avoir un développement végétatif normal. L'impact sur les rendements varie de 20 à $50 \%$. Le nombre de ligneux qui poussent le long des ouvrages traduit la régénération favorisée par les aménagements. Dans la zone d'intervention du projet PATECORE, ce nombre est en moyenne de 11 pieds pour $100 \mathrm{~m}$ de cordons pierreux.

- Le zaï : il consiste à creuser des trous larges de 20 à $30 \mathrm{~cm}$ de diamètre et de $15 \mathrm{~cm}$ de profondeur, dans lesquels on dépose du compost ou de la poudrette issue des déjections des animaux et des ordures ménagères. Les termites, attirés par la matière organique, creusent des galeries au fond des cuvettes qu'ils transforment en entonnoirs. Les eaux de ruissellement y pénètrent, créant des poches d'humidité en profondeur, à l'abri de l'évaporation, ce qui entraîne une amélioration des propriétés hydrodynamiques du sol. L'effet conjugué de la matière organique et de l'humidité permet une meilleure implantation des semis. C'est pourquoi les re-semis et les conséquences des accès précoces de sécheresse sont souvent évités. Les rendements augmentent de façon spectaculaire (50 à plus de $100 \%$ ). Répété plusieurs années sur le même sol, le zaï permet de reconstituer la fertilité du sol et le couvert végétal. L'inventaire des adventices réalisé par Trouillier (2003) fait ressortir l'apparition de plusieurs espèces herbacées sur les parcelles aménagées, tandis que les parcelles témoins restent nues. Lors de la confection des trous, les paysans doivent tenir compte de certains facteurs, tels que la toposéquence et l'écartement (selon qu'ils envisagent ou non d'utiliser la traction animale). Le zaï est généralement pratiqué sur les sols gravillonnaires, mais il est de plus en plus généralisé sur tous les types de sol pour minimiser le déficit hydrique. Le zaï exige beaucoup de main-d'œuvre.

- Les demi-lunes : ce sont des cuvettes en demi-cercle creusées et ceinturées avec les déblais disposés en arc de cercle ouvert à l'amont. Le creux recueille l'eau piégée par les bras de la demi-lune. L'amont du creux sert d'impluvium. L'association entre un impluvium inculte qui reçoit l'eau de pluie et la partie creusée et travaillée où elle est retenue constitue les éléments principaux de ce dispositif. Les demi-lunes sont généralement disposées en quinconce sur les versants, de façon à retenir toutes les eaux de ruissellement ; cela permet aux plantes cultivées dans la demi-lune de recevoir beaucoup plus d'eau que ne lui apportent directement les pluies. Les demi-lunes entraînent non seulement l'accroissement des rendements mais également l'apparition d'un couvert végétal. L'apport de matière organique facilite le développement $d$ 'une flore importante. La demi-lune peut avoir un rayon 
Photo 1. Les différentes technologies de lutte contre la désertification.
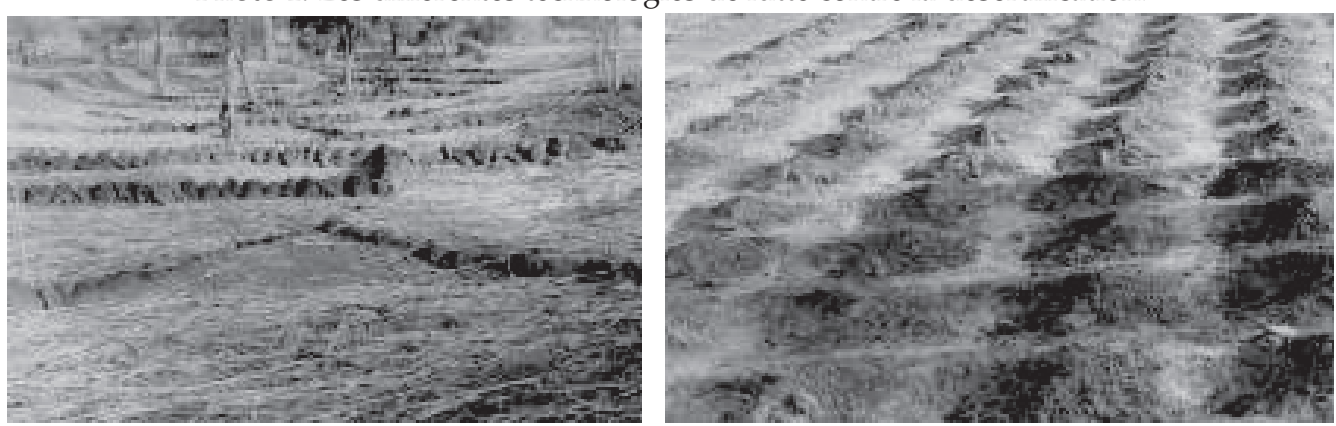

Cordons pierreux
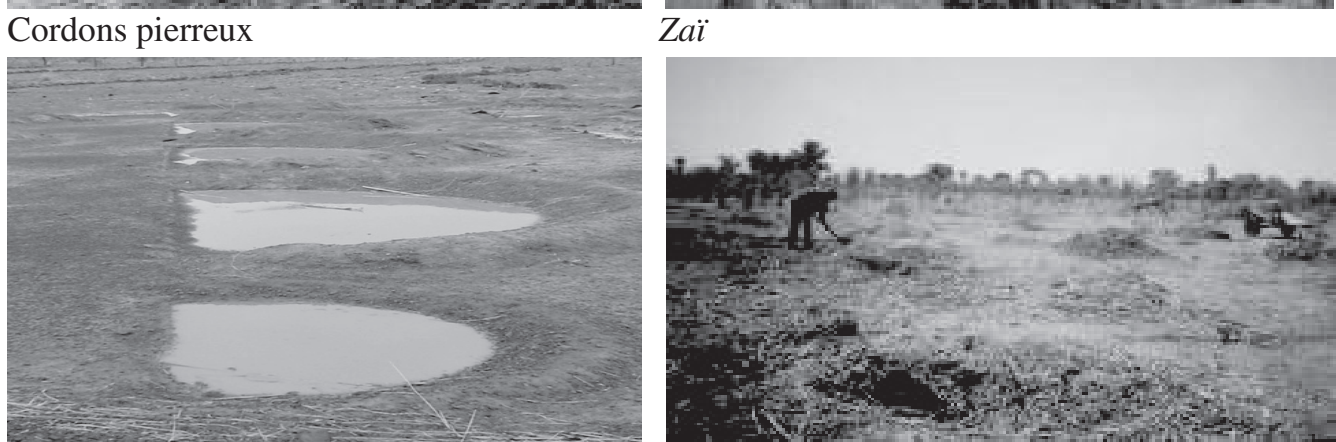

Demi-lunes (photo IFDC)
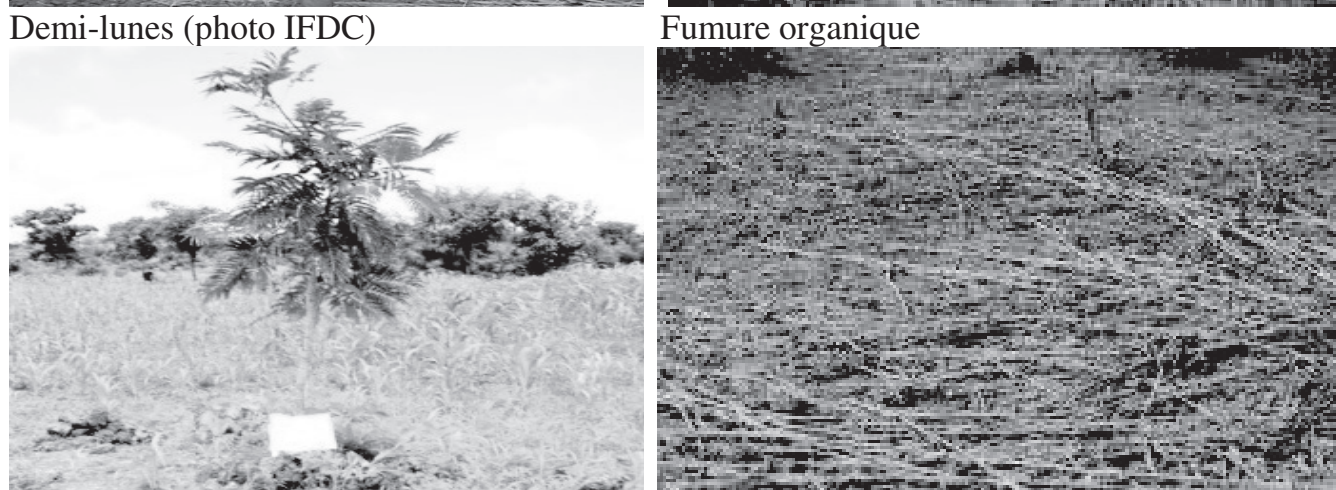

Régénération naturelle assistée

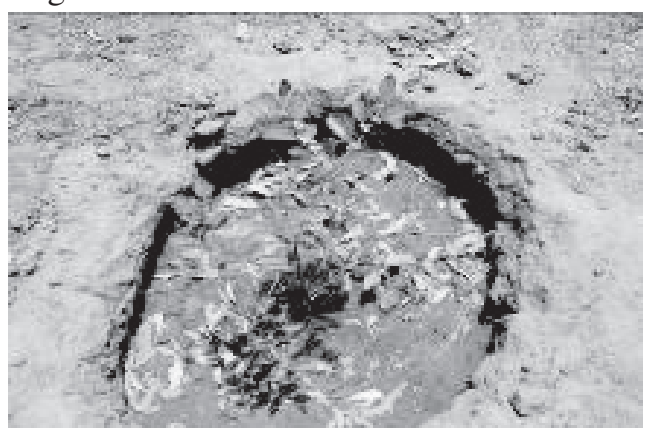

Plantation d'arbres

Paillage
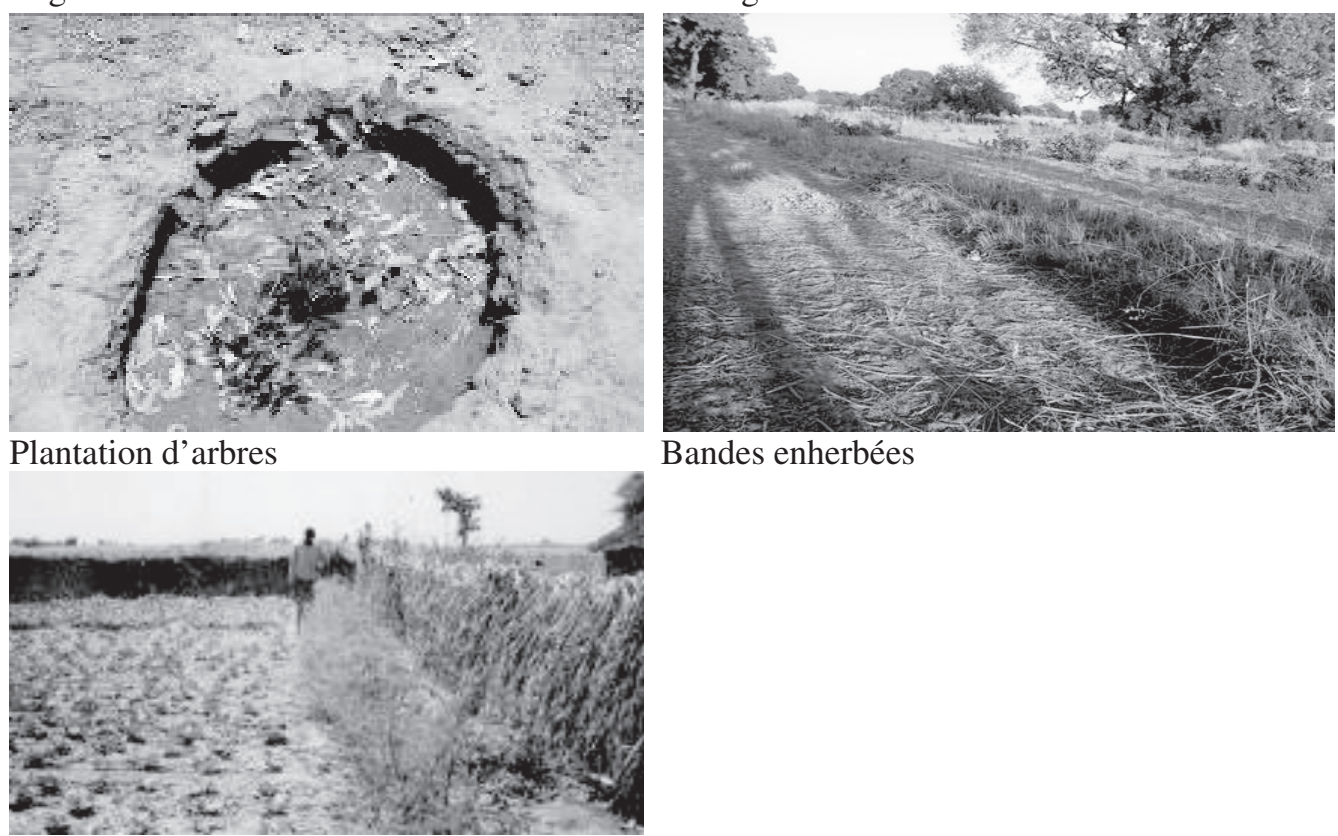

Bandes enherbées

Haies vives 
allant de 2 à $3 \mathrm{~m}$ et une profondeur de 30 à $40 \mathrm{~cm}$. Tout comme le zaï, la confection des demi-lunes est un gros labeur et demande beaucoup de main-d'œuvre.

- L'épandage de fumure organique : l'utilisation de la fumure organique constitue l'une des plus vieilles méthodes d'amélioration de la fertilité du sol. Elle s'effectue par le biais de plusieurs procédés (déchets domestiques, déjections animales, compost, résidus de récolte). La fumure organique est un amendement qui joue un rôle différent de celui des engrais minéraux. Une fois appliquée, la fumure organique libère progressivement les nutriments qu'elle contient tout en améliorant les propriétés physiques, chimiques et biologiques du sol. Elle permet de résoudre à long terme les problèmes de fertilité. L'impact sur les rendements est de 15 à 50 \% sur des sols de qualité médiocre (Dugué, 1989). Cependant, ils peuvent doubler sur des sols de bonne qualité.

- La régénération naturelle assistée (RNA) : conseillée par les services étatiques (Agriculture, Eaux et Forêts) , elle consiste à épargner certains arbustes (arbres utiles et à usages multiples), de façon à reconstituer un couvert ligneux sur les champs. Selon les producteurs, un certain taux de présence d'arbres sur les champs contribue à freiner l'écoulement de l'eau, donc à réduire l'érosion tout en permettant une restauration de la diversité biologique.

- Le paillage : il consiste à recouvrir le sol d'une couche de $2 \mathrm{~cm}$ d'herbes, équivalant de 3 à 6 t/ha, de branchages ou encore de résidus culturaux (tiges de mil ou de sorgho), de façon à stimuler l'activité des termites. Ces derniers vont casser la croûte superficielle $\mathrm{du}$ sol en creusant des galeries sous les paillis. Il en résulte un ameublissement du sol et une augmentation de sa porosité qui permettent une meilleure infiltration de l'eau (Zombré et al., 2000). L'application du paillis dans les zones semi-arides du Sahel, où l'érosion éolienne est présente, entraîne une accumulation de particules sous forme de sédiments sous les paillis (Mando et Stroosnijder, 1999). Le paillage entraîne également la réhabilitation de la végétation dès la première année d'application (Mando et Brussard, 1999) et un développement de végétation couvrant complètement un sol nu dans un délai de deux ans. Cette performance de la végétation sous paillis est la réponse de celle-ci à l'effet du paillage sur l'amélioration de la structure du sol et sur la disponibilité en eau et en nutriments dans le sol.

- La plantation d'arbres : il s'agit surtout d'arbres fruitiers. Selon la coutume, ces plantations consacrent l'appropriation effective et individuelle de la terre.

- Les bandes enherbées : ce sont des bandes de végétation permanentes d'herbes et d'arbustes établies le long des courbes de niveau dans les champs. Disposées perpendiculairement au sens des écoulements
Tableau 2. Coûts d'adoption (en FCFA/ha) des technologies de lutte contre la désertification.

\begin{tabular}{lcc}
\hline Technologie & $\begin{array}{c}\text { Coût par ha } \\
\text { (FCFA) }\end{array}$ & $\begin{array}{c}\text { Part prise en charge } \\
\text { par le paysan (en \%) }\end{array}$ \\
\hline Cordons pierreux & 38000 à 112 000 & 30 à 85 \\
Zaï & 723000 & 98 \\
Demi-lune & 84000 à 322000 & 89 à 97 \\
Fumure organique & 12500 à 20000 & 100 \\
Régénération naturelle assistée & nd & nd \\
Paillage & nd & 100 \\
Plantation d'arbres & 62000 à 100000 & 100 \\
Bande enherbée & nd & nd \\
Haie vive & nd & nd \\
\hline
\end{tabular}

Sources : compilations à partir d'études diverses (PSB/PB, PATECORE, PDRD, FNGN).

nd $=$ non disponible.

diffus et suivant des critères adéquats liés aux sols, aux pentes, à l'occupation du sol et aux pluies, les bandes enherbées favorisent trois types de processus :

- le ralentissement du ruissellement diffus de surface qui les traverse, du fait de la rugosité de surface importante de la végétation de la bande enherbée ;

- la diminution éventuelle de ce flux de ruissellement diffus, par infiltration accrue due à la présence d'une végétation dense ;

- le dépôt de sédiments du fait des deux processus précédents.

Les bandes enherbées jouent un rôle important pour les terrains drainés et ne trouvent leur pleine efficacité qu'en fonction de la topographie des lieux et de la nature des sols. Naturellement, leur efficacité dépend aussi de leur largeur.

- La haie vive : elle est traditionnellement utilisée pour délimiter des parcelles ou des jardins, généralement de petite superficie. Elle joue un rôle de brise-vent. Elle contribue aussi à la conservation et à la restauration des sols en ralentissant le ruissellement des eaux de pluie et en réduisant l'érosion.

De cette description, on peut retenir que les cordons pierreux, le zaï et les demi-lunes sont exigeants en investissement humain (force de travail), mais sont préférés par les paysans aux autres techniques à cause de leur impact sur le rendement et sur le milieu. Leur mise en œuvre engendre des coûts assez élevés (Tab. 2). Ces coûts intègrent à la fois la main-d'œuvre familiale, le petit matériel, le transport des substrats. Comme il s'agit surtout d'investissement à base de travail, c'est la main-d'œuvre familiale qui constitue souvent l'essentiel des coûts ${ }^{4}$. À elle seule, elle peut représenter plus de 50 à $90 \%$ des coûts $\mathrm{d}$ 'adoption. Ce sont donc des investissements énormes et

\footnotetext{
${ }^{4}$ Le paysan a souvent l'illusion d'un travail gratuit. Pour lui, ce qui ne lui coûte que du travail ne coûte rien.
} 
Tableau 3. Techniques de lutte contre la désertification et proportion (\%) des ménages qui les pratiquent en fonction du statut foncier.

\begin{tabular}{|c|c|c|c|c|c|c|c|c|c|c|c|c|}
\hline \multirow[t]{2}{*}{ Technologie } & \multicolumn{2}{|c|}{ Kirsi } & \multicolumn{2}{|c|}{ Boulcon } & \multicolumn{2}{|c|}{ Dafiré } & \multicolumn{2}{|c|}{ Sakou } & \multicolumn{2}{|c|}{ Dossi } & \multicolumn{2}{|c|}{ Bahoun } \\
\hline & PF & NPF & PF & NPF & PF & NPF & PF & NPF & PF & NPF & PF & NPF \\
\hline Cordons pierreux & 100 & 94 & 100 & 88,9 & 100 & 95,2 & 100 & 95,2 & 80 & 19 & 0 & 0 \\
\hline Zä̈ & 100 & 100 & 50 & 75 & 83,3 & 76,2 & 89,5 & 76,2 & 0 & 0 & 0 & 0 \\
\hline Demi-lune & 17,4 & 6 & 25 & 36,1 & 0 & 0 & 0 & 0 & 0 & 0 & 0 & 0 \\
\hline RNA & 91,3 & 75,5 & 0 & 16,7 & 0 & 0 & 42,1 & 23,8 & 20 & 9,5 & 0 & 0 \\
\hline Plantation d'arbres & 87 & 47,1 & 75 & 58,3 & 11,1 & 9,5 & 52,6 & 28,6 & 40 & 0 & 0 & 0 \\
\hline Bandes enherbées & 26,1 & 17,6 & 0 & 2,8 & 44,4 & 42,9 & 42,1 & 52,4 & 0 & 4,8 & 10 & 0 \\
\hline Paillage & 91,3 & 82,4 & 0 & 5,6 & 0 & 4,8 & 36,8 & 4,8 & 40 & 4,8 & 0 & 4,8 \\
\hline Fumure organique & 100 & 100 & 75 & 75 & 33,3 & 19 & 26,3 & 14,3 & 100 & 100 & 100 & 95,2 \\
\hline Haie vive & 4,3 & 5,9 & 0 & 0 & 0 & 0 & 5,3 & 0 & 30 & 4,8 & 0 & 0 \\
\hline
\end{tabular}

$\mathrm{PF}=$ possesseur foncier.

$\mathrm{NPF}=$ non-possesseur foncier.

RNA = régénération naturelle assistée.

de long terme que les producteurs ne peuvent consentir que s'ils ont l'assurance d'en profiter.

\section{Statut foncier et pratique des techniques de lutte contre la désertification}

Selon la coutume, les non-possesseurs fonciers n'avaient pas le droit de planter des arbres ou d'entreprendre des améliorations qui pourraient leur conférer une prééminence sur la terre. En effet, l'entreprise d'une amélioration a été souvent interprétée comme une affirmation par l'étranger à un droit permanent et elle s'est, de ce fait, souvent heurtée à une résistance des possesseurs fonciers. Ces restrictions ont conduit à une exploitation minière des terres. En effet, le système coutumier d'exploitation des terres encourage les ménages à les exploiter jusqu'au degré maximal possible, mais ne les pousse guère à les entretenir ou à les améliorer. Cependant, il semble que les transformations foncières, sociales et économiques en cours dans le milieu rural aient préparé les conditions d'une élimination progressive des différentes restrictions coutumières.

\section{Une adoption diversifiée des techniques anti-désertification}

Le choix de telle technique dépend des types de sol et des moyens matériel et humain disponibles (Roose, 1994, p. 286-288; Rochette, 1989, p. 155, 248, 344 pour la répartition des aménagements en fonction de la toposéquence). Dans un même champ, plusieurs techniques peuvent être appliquées, soit individuellement, soit en association. Le tableau 3 donne la proportion des producteurs qui pratiquent ces différentes techniques. On observe une distinction nette entre les villages du Plateau central et ceux de l'Ouest. Dans le Plateau central, presque toutes les techniques sont pratiquées, souvent de manière combinée les unes avec les autres. Cela n'est pas le cas des villages de l'Ouest, qui ont surtout adopté les cordons pierreux et la fumure organique.

Dans les villages du Plateau central, aussi bien les possesseurs fonciers que les non-possesseurs ont recours aux cordons pierreux, au zaï et à l'épandage de fumure organique. L'engouement des populations pour ces techniques s'explique non seulement par leur impact sur les rendements et le milieu naturel, mais également par le soutien (matériel et financier) accordé par les projets de développement et les ONG pour leur réalisation. C'est à la faveur de la sécheresse des années 1970 que ces derniers se sont investis dans le Plateau central pour limiter la dégradation et garantir une production agricole acceptable. En tirant des leçons des échecs des grands projets de défense et de restauration des sols des années 1960, ils ont impliqué les populations dans leurs actions par la sensibilisation, la formation et le soutien matériel et financier. Viennent ensuite les plantations d'arbres, la régénération naturelle assistée et les bandes enherbées. La haie vive est la moins utilisée.

Dans l'Ouest, l'ampleur des techniques de lutte contre la désertification est encore faible. Trois techniques sont utilisées : l'épandage du fumier, les cordons pierreux et le paillage. Dans les deux villages, la presque totalité des ménages recourt à la fumure organique pour l'amélioration de la fertilité du sol.

Les résultats du tableau 3 suggèrent que les techniques de lutte contre la désertification sont plus diversifiées sur le Plateau central (Kirsi, Boulcon, Dafiré, Sakou) que dans l'Ouest (Dossi, Bahoun). Cette situation s'explique par le degré critique de la dégradation des terres sur le Plateau central. Par contre, il n'y a pas de dichotomie nette entre les techniques pratiquées par les possesseurs terriens et celles des non-possesseurs. Ceci implique-t-il l'inexistence de contraintes à la mise en 
œuvre des techniques de lutte contre la désertification, ou évolue-t-on vers la transformation des droits d'usage en droits de propriété au fil des ans?

\section{Perceptions des contraintes foncières par les acteurs locaux}

Le statut foncier actuel permet-il aux producteurs, en particulier aux non-possesseurs fonciers, $d$ 'investir dans la préservation des ressources naturelles? Les résultats de l'enquête indiquent qu'un consensus se dégage en ce qui concerne les possesseurs fonciers. Tous considèrent (à presque $100 \%$ ) qu'il n'existe plus de contrainte à la réalisation des techniques de lutte contre la désertification. La tendance est identique pour les non-possesseurs terriens, qui estiment également qu'il n'y a plus de contrainte, exception faite de la plantation d'arbres, mais à condition d'en informer le possesseur auparavant (plus de $80 \%$ des producteurs par village). Cette information préalable du possesseur est surtout une formalité. Elle relève des relations bilatérales, familiales ou de dépendance entre les acteurs, dont la qualité dépend beaucoup du respect des engagements pris. Les non-possesseurs savent tous que, pour ce qui concerne les dons et les prêts à long terme, même si les possesseurs continuent d'exercer un contrôle social, ceux-ci ne peuvent plus reprendre leur terre que si le bénéficiaire s'est rendu coupable d'une faute grave. Dans le cas contraire, une pression sociale s'exercerait sur eux. Cela confère aux non-possesseurs une certaine assurance, tout en les incitant à maintenir de bons rapports avec les possesseurs.

Ces résultats sont corroborés par ceux obtenus au nord-ouest du Plateau central. En effet, dans les villages de Lankoé et de Kiembara, respectivement $88 \%$ et $56 \%$ des ménages affirment ne plus subir de restriction dans les activités qu'ils peuvent conduire dans les champs, que ceux-ci aient été acquis par emprunt ou par donation (Ouédraogo et al. 2002). Stamm et al. (2003), dans leur étude sur les micro-politiques foncières dans les villages de la province du Bam au Burkina Faso, indiquent que 92 à $98 \%$ des personnes interrogées considèrent que les producteurs sont libres de gérer et d'aménager les terres qu'ils exploitent. Seulement 2,4 à $8 \%$ des producteurs sont réticents en ce qui concerne la plantation d'arbres. Des résultats similaires ont également été obtenus par Banderé et Batta (1999) dans l'Est du Burkina Faso. En effet, ces derniers affirment que la question foncière n'intervient plus dans la décision du ménage d'investir dans les techniques de conservation des eaux et des sols. Ils mentionnent «que les ménages qui exploitent des parcelles empruntées ne sont pas inquiétés, les possesseurs ne les réclamant pas lorsqu'elles sont mises en valeur ». À Daboura (village de la région ouest), les autochtones encouragent les migrants à entreprendre des améliorations foncières, possesseurs et non-possesseurs terriens coopérant pour sauvegarder le potentiel de production de la terre (Samandoulogou, 2000). Ces résultats contrastent cependant avec les affirmations de Kessler et al. (1995), selon lesquelles il est impossible de prévoir le temps que le paysan non-possesseur peut rester sur une parcelle au Burkina Faso.

Ce qui ressort également des résultats de nos enquêtes, c'est la différence de perception des producteurs entre les améliorations foncières et la plantation d'arbres. Si les premières sont acceptées par la quasi-totalité des producteurs, la seconde semble poser encore des problèmes. En effet, il existe une contradiction entre possesseurs et non-possesseurs fonciers sur la question. Si la grande majorité des premiers affirment que la plantation est autorisée, les seconds considèrent que des entraves existent encore. Ils sont plus nombreux dans les villages du Plateau central que dans l'Ouest $(53 \%$ à Kirsi et $67 \%$ à Dafiré affirment que la plantation d'arbres ne leur est pas autorisée, contre $24 \%$ à Dossi et $48 \%$ à Bahoun). Cette contradiction témoigne des blocages ou des réticences qui persistent encore de la part des possesseurs fonciers. En effet, la plantation d'arbres a toujours été considérée comme une tentative d'appropriation définitive parce qu'elle mobilise la terre pour une longue période. Elle traduit la crainte des possesseurs de perdre le contrôle de cette terre.

Il semble donc que les évolutions récentes aient conduit les paysans à différencier améliorations foncières et plantation d'arbres. Si la première technique conduit à une augmentation du potentiel de production de la terre, la seconde est considérée comme un moyen de matérialisation de l'appropriation de la terre et, par conséquent, elle reste difficilement acceptée.

\section{Conclusion : la levée du blocage foncier}

Bien que le régime foncier coutumier soit encore le système dominant de gestion des ressources dans les sociétés du Burkina Faso, la combinaison de la croissance démographique, des changements techniques, économiques, climatiques et politiques a entraîné une évolution des institutions. D'une manière générale, il semble que les techniques de lutte contre la désertification soient acceptées par tous les ménages des villages enquêtés (exception faite de la plantation d'arbres). Ce besoin d'investir dans la réhabilitation des ressources naturelles s'expliquerait par la nécessité de maintenir ou d'accroître la productivité d'une terre qui s'est appauvrie, suite à une exploitation continue non soutenue par des actions de restauration. Il semble donc que la pression démographique et les techniques culturales inadaptées qui ont engendré la dégradation des ressources naturelles aient créé un besoin d'investissement en matière de potentiel 
agricole, indépendamment du statut foncier. Dans certains cas, ce sont la disparition de la jachère et les difficultés d'acquisition des engrais minéraux qui ont obligé les producteurs à s'investir dans les améliorations agraires. Présentement, il est de l'intérêt à la fois des possesseurs et des non-possesseurs fonciers d'inverser la tendance à la dégradation des terres, s'ils ne veulent pas compromettre à long terme l'avenir de la production agricole.

Les résultats des enquêtes suggèrent donc une évolution positive par rapport à la situation d'antan où le système foncier n'autorisait pas les investissements, particulièrement de la part des non-possesseurs terriens. Les arrangements fonciers sont ainsi loin d'être statiques; ils ont été assez souples pour permettre des adaptations (Lavigne Delville et al., 2001). De nos jours, ils sont en train d'évoluer sous la combinaison de la pression de la population, de la dégradation des ressources naturelles, de l'économie monétaire et de l'individualisme économique. On peut conclure qu'en ce qui concerne l'acceptation des techniques de lutte contre la désertification, les modes coutumiers d'accès à la terre ne jouent plus tellement un rôle de blocage social. Le système foncier coutumier a été suffisamment souple pour permettre des ajustements des systèmes de production face aux changements socioéconomiques.

\section{Références}

Banderé, P.J., Batta F., 1999. Conservation des eaux et des sols au Burkina Faso. Revue documentaire de l'ONG Voisins mondiaux, siège pour l'Afrique de l'Ouest : Ouagadougou, Burkina Faso, et de Overseas Development Institute, Londres.

Bassett, T.J., 1993. Introduction : The land question and agricultural transformation in Sub-Saharan Africa, in Bassett, T.J., Crummey, D.E. (Eds), Land in African Agrarian Systems, Madison, University of Wisconsin Press, 3-31.

Biaou, G., 1991. Régime foncier et gestion des exploitations agricoles sur le plateau Adja (Bénin). Thèse de doctorat de $3^{\mathrm{e}}$ cycle, CIRES, Université nationale de Côte d'Ivoire, Abidjan.

Binswanger, H.P., Deininger, K., Feder, G., 1995. Power, distortions, revolt and reform in agricultural land relations, in Behrman, J., Srinivasan, T.N. (Eds), Handbook of Development Economics, Amsterdam, North-Holland, 3B, 2659-2772.

Boutillier, J.-L., 1964. Les Structures foncières en Haute-Volta, Ouagadougou, Centre IFAN- ORSTOM.

Bruce, J., 1985. Land Tenure Issue in Project Design: Strategy for Development in Sub-Saharan Africa, Madison, Land Tenure Center.

Bruce, J.W., Migot-Adholla, S.E. (Eds), 1994. Searching for Land Tenure Security in Africa, Dubuque, Kendal/Humt.

Curie, J.M., 1981. The Theory of Agricultural Land Tenure, Cambridge, Cambridge University Press.

Dugué, P., 1989. Possibilités et limites de l'intensification des systèmes de culture vivriers en zone soudano-sahélienne: le cas du Yatenga. Thèse de docteur ingénieur, ENSA, Montpellier.

Feder, G., 1987. Land ownership security and farm productivity : evidence from Thailand, Journal of Development Studies, 24,1, 16-30.
Feder, G., Feeny, D., 1991. Land tenure and property rights : theory and implication for development policy, The World Bank Economic Review, 5, 1, 135-153.

Francis, P., 1986. Nationalisation des terres et régime foncier dans le sud du Nigeria, Bulletin du CIPEA, 24, 2-7.

Kessler, C.A., Spaan, W.P., Van Driel, W.F., 1995. Choix et modalités d'exécution des mesures de conservation des eaux et des sols au Sahel: une comparaison de cinq projets de développement, Wageningen, WAU.

Kohler, J.-M., 1971. Activités agricoles et changements sociaux dans l'Ouest-Mossi (Haute-Volta), Ouagadougou, ORSTOM.

Lavigne Delville, P., Toulmin, C., Colin, J.-P., Chauveau, J.-P., 2001. Sécurisation des droits fonciers délégués en Afrique de l'Ouest. Dossier 107, programme Zones arides, IIED, Londres.

Mando, A., Brussard, L., 1999. The role of termite in the breakdown of straw under Sahelian condition, Biological and Fertility of Soil, 9, 332-334.

Mando, A., Stroosnijder, L., 1999. The biological and physical role of much in the rehabilitation of crusted soil in the Sahel, Land Use and Management, 15, 123-130.

Marchal, J.-Y., 1986. Vingt ans de lutte antiérosive au nord du Burkina Faso, Cahiers ORSTOM, série Pédologie, 22, 2, 173180.

Mathieu, P., 1995. Le foncier et la gestion des ressources naturelles, in Mathieu, P., Laurent, P.J. (Eds), Actions locales, enjeux fonciers et gestion de l'environnement au Sahel, Cahiers du CIDEP, 27, 46-59.

Mellor, J.W., 1966. The Economics of Agricultural Development, London, Cornell University Press.

Mesnil, J., 1970. La Connaissance du milieu et vulgarisation agricole : le cas de l'opération Centre Mossi, Paris, Société d'aide technique et de coopération, III.

Migot-Adholla, S.E., Hazell, P.B., Blarel, B., Place, F., 1993. Indigenous land right systems in sub-Saharan Africa, a constraint on Productivity?, in Hoff, K., Braverman, A., Stiglitz, J. (Eds), The Economics of Rural Organisation, Oxford, Oxford University Press / World Bank, 269-291.

Ouédraogo, R.S., Sawadogo, J.P., Stamm, V., Thiombiano, T., 1996. Tenure, agricultural pratices and land productivity in Burkina Faso : some recent results, Land Use Policy, 13, 3, 229-232.

Ouédraogo, S., 1991. Influence des modes d'accès à la terre sur la productivité des exploitations agricoles : le cas de la zone ouest du Burkina Faso. Thèse de doctorat de $3^{\mathrm{e}}$ cycle, CIRES, Université nationale de Côte d'Ivoire, Abidjan.

Ouédraogo S., 1993. Quel(s) régime(s) foncier(s) pour les aménagements hydro-agricoles?, in Quel environnement pour le développement de l'irrigation au Burkina Faso? Actes du séminaire atelier, Ouagadougou, Ministère de l'Eau.

Ouédraogo, S., Millogo, M.C.S., Lalba, A., Troré, J.-N., Bonkoungou, J., 2002. Participation des producteurs à la mise en cuvre des projets de lutte contre l'érosion : un modèle d'intervention sociale plus approprié, Ouagadougou, INERA.

Platteau, J.-P., 1996. The evolutionary theory of land rights as applied to Sub-Saharan Africa: a critical assessment, Development and Change, 27,1, 29-86.

Platteau, J.-P., 1998. Une analyse des théories évolutionnistes, des droits sur la terre, in Lavigne Delville, P. (Ed.), Quelles politiques foncières pour l'Afrique rurale?, Paris, Karthala, 123130.

Reij, C., 1983. L'Évolution de la lutte anti-érosive en Haute-Volta depuis les indépendances : vers une plus grande participation 
de la population, Amsterdam, Institute for Environmental Studies, Free University.

Rochette, R.M., 1989. Le Sahel en lutte contre la désertification : leçons d'expériences, Weikersheim, Margraf.

Roose, E.J., 1994. Introduction à la gestion conservatoire de l'eau, de la biomasse et de la fertilité des sols (GCES), Bulletin pédologique de la FAO, 70.

Runge, C.F., 1992. Common property and collective action in economic development, in Bromley, W.D. (Ed.), Making the Commons Work: Theory, Practice and Policy, San Francisco, ICS Press, 17-39.

Samandoulogou, Y., 2000. Sécurité foncière et facteurs déterminant la décision d'investissement des exploitations de migrants dans la zone ouest du Burkina Faso : le cas de Daboura. Mémoire de fin d'étude, IPR-IFRA, Université du Mali, Bamako.

Stamm, V., Sawadogo, J.-P., Ouédraogo, S.R., Ouédraogo, D., 2003. Micro-politiques foncières dans trois villages de la province

Reçu le 22 août 2005. Accepté le $1^{\text {er }}$ février 2007. du Bam au Burkina Faso: stratégies locales d'échange de terres. Dossier 124, programme Zones arides, IIED, Londres.

Sjaastad, E., Bromley, W.D., 1997. Indigenous land rights in subSaharan African: appropriation security and investment demand, World Development, 25, 4, 549-562.

Trouillier, A., 2003. Réhabilitation de la fertilité des sols au Burkina Faso : approche agronomique et environnementale comparative du système zaï. Mémoire INA P-G/IRD, Paris.

Vlaar, J.C.J., 1992. Les Techniques de conservation des eaux et des sols dans les pays du Sahel, Ouagadougou, CIEH.

Zombré, N.P., Mando, A., Ilboudo, J.-B., 2000. Impact des conservations des eaux et des sols sur la restauration des jachères très dégradées au Burkina Faso, in Pontanier, R., Floret, C. (Eds), La Jachère en Afrique tropicale : rôles, aménagement, alternatives, Paris, IRD Éditions/John Libbey Eurotext, I, 771-777.

To access this journal online: www.edpsciences.org 